\title{
HOT CORE, OUTFLOWS, AND MAGNETIC FIELDS IN W43-MM1 (G30.79 FIR 10)
}

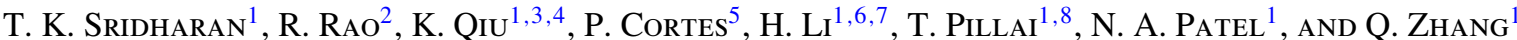 \\ ${ }^{1}$ Harvard-Smithsonian Center for Astrophysics, 60 Garden Street, Cambridge, MA 02138, USA; tksridha@ cfa.harvard.edu \\ ${ }^{2}$ Submillimeter Array, Academia Sinica Institute of Astronomy and Astrophysics, 645 N. Aohoku Place, Hilo, HI 96720, USA \\ ${ }^{3}$ Max Planck Institute for Radioastronomy, Bonn 53121, Germany \\ ${ }^{4}$ School of Astronomy and Space Science, Nanjing University, Nanjing 210093, China \\ ${ }^{5}$ National Radio Astronomy Observatory-Joint ALMA Office, Alonso de Cordova 3107, Vitacura, Santiago, Chile \\ ${ }^{6}$ Max Planck Institute for Astronomy, Hiedelberg, Germany \\ ${ }^{7}$ Department of Physics, The Chinese University of Hong Kong, Hong Kong \\ ${ }^{8}$ Astronomy Department, California Institute of Technology, 1200 East California Boulevard, Pasadena, CA 91125, USA \\ Received 2013 November 8; accepted 2014 January 27; published 2014 February 26
}

\begin{abstract}
We present submillimeter spectral line and dust continuum polarization observations of a remarkable hot core and multiple outflows in the high-mass, star-forming region W43-MM1 (G30.79 FIR 10), obtained using the Submillimeter Array. A temperature of $\sim 400 \mathrm{~K}$ is estimated for the hot core using $\mathrm{CH}_{3} \mathrm{CN}(J=19-18)$ lines, with detections of $11 \mathrm{~K}$-ladder components. The high temperature and the mass estimates for the outflows indicate high-mass star formation. The continuum polarization pattern shows an ordered distribution, and its orientation over the main outflow appears to be aligned with the outflow. The derived magnetic field indicates slightly supercritical conditions. While the magnetic and outflow energies are comparable, the $B$-field orientation appears to have changed from parsec scales to $\sim 0.1 \mathrm{pc}$ scales during the core/star formation process.
\end{abstract}

Key words: ISM: jets and outflows - ISM: magnetic fields - stars: formation - stars: massive

Online-only material: color figures

\section{INTRODUCTION}

The process of high-mass star formation and early evolution is marked by the phenomena of outflows (e.g., Shepherd \& Churchwell, 1996; Beuther et al. 2002; Zhang et al. 2005) and hot cores (e.g., Cesaroni et al. 1992; Cesaroni et al. 2010). Precious little is known about the role of magnetic fields in the process (e.g., DR21(OH): Lai et al. 2003; Girart et al. 2013; G34.41: Cortes et al. 2008; G31.41: Girart et al. 2009; IRAS18089-1732: Beuther et al. 2010; W51, G5.89: Tang et al. 2009a, 2009b, 2013). Due to the large distances to highmass, star-forming regions, interferometric studies are critical to understanding them better, as in the above examples. It is also necessary to study regions in early stages of evolution, preferably the high-mass proto-stellar object phase (HMPOs; e.g., Sridharan et al. 2002) or younger, to probe pristine conditions. In this Letter, we report the discovery of a high temperature hot core and multiple outflows toward an early stage object, W43-MM1 (G30.79 FIR10), and the magnetic field distribution around it, using submillimeter wavelength spectral line and continuum polarization observations at $345 \mathrm{GHz}$, obtained with the Submillimeter Array ${ }^{9,10}$ (SMA; Ho et al. 2004).

\section{OBJECT AND OBSERVATIONS}

W43-MM1, also known as G30.79 FIR 10, is the brightest dust emission core in the W43 "mini-starburst" region (Motte et al. 2003; Bally et al. 2010). The region harbors UC-H II

\footnotetext{
9 The SMA is a joint project between the Smithsonian Astrophysical Observatory and the Academia Sinica Institute of Astronomy and

Astrophysics, and is funded by the Smithsonian Institution and the Academia Sinica.

10 The National Radio Astronomy Observatory is a facility of the National Science Foundation operated under cooperative agreement by Associated Universities, Inc.
}

regions, water masers, and methanol masers, marking highmass star formation. The FIR10/MM1 core is at the head of a cometary infrared dark cloud located at $5.5 \mathrm{kpc}$ (from maser parallax; Zhang et al. 2014), facing a giant $\mathrm{H}$ II region powered by a cluster of WR-OB stars. It is devoid of centimeter-wavelength emission, which may suggest an early, HMPO evolutionary stage. In addition, evidence for infall in multiple spectral lines and high accretion rates, and extended $\mathrm{SiO}$ emission possibly due to colliding outflows have also been reported (Cortes et al. 2010; Cortes 2011; Herpin et al. 2012; Nguyen-Lu'o'ng et al. 2013), making the region very interesting. Polarized dust emission at $1.3 \mathrm{~mm}$ suggested an inconclusive pinched morphology for the magnetic field (Cortes \& Crutcher 2006), with a field strength of $1.7 \mathrm{mG}$, implying a near critical mass-to-magnetic flux ratio of 0.9 , later refined to 1.9 (Cortes 2011). If magnetic support is dominant in the natal core, a pinched morphology is expected.

The SMA observations reported here, intended to better delineate the $B$ field and to study line emission from the core, were obtained in the sub-compact and compact configurations. The observations were conducted on 2007 April 25 and 2008 May 28 under excellent weather conditions with a $225 \mathrm{GHz}$ zenith opacity of $\sim 0.05$. The sub-compact observations only had five antennas. The receivers were tuned to 346.51 and $349.42 \mathrm{GHz}$ at the center of the upper side band for the two observations with the phase center at $(\alpha, \delta)=18: 47: 47.0$, $-01: 54: 30.0$ (J2000). The correlator was configured to provide a uniform resolution of $0.81 \mathrm{MHz}\left(0.7 \mathrm{~km} \mathrm{~s}^{-1}\right.$ at $\left.349 \mathrm{GHz}\right)$. The combined data have a UV coverage of $16-160 k \lambda$. The polarization, phase, and flux calibrators were 3c273, 1751+096, and Uranus, respectively. Standard data reduction procedures were used under MIRIAD. A discussion of the SMA polarimetry system can be found in Marrone et al. (2006) and Marrone \& Rao (2008). For continuum, multi-frequency synthesis was used to combine the two data sets with slightly differing frequencies, by $3 \mathrm{GHz}$, for the two tracks. 
Table 1

Continuum Emission

\begin{tabular}{|c|c|c|c|c|c|c|c|c|c|}
\hline ID & $\begin{array}{c}\text { R.A. } \\
\text { (J2000.0) }\end{array}$ & $\begin{array}{c}\text { Decl. } \\
\text { (J2000.0) }\end{array}$ & $\begin{array}{c}\Delta \text { R.A., } \Delta \text { Decl. } \\
(\prime \prime)\end{array}$ & $\begin{array}{l}\text { Peak, Err } \\
\left(\mathrm{Jy} \mathrm{bm}^{-1}\right)\end{array}$ & $\begin{array}{l}\text { Intg. } \\
\text { (Jy) }\end{array}$ & $\begin{array}{l}\text { Maj } \\
(\prime \prime)\end{array}$ & $\begin{array}{l}\text { Min } \\
(/ \prime)\end{array}$ & $\begin{array}{l}\text { P.A. } \\
\text { (Deg.) }\end{array}$ & $\begin{array}{l}\text { Mass } \\
\left(M_{\odot}\right)\end{array}$ \\
\hline $\bar{A}$ & $18: 47: 47.00$ & $-1: 54: 26.6$ & 0.10 .1 & $7.9,0.61$ & 11.5 & 1.7 & 1.3 & -52 & $920-80$ \\
\hline B & $18: 47: 46.86$ & $-1: 54: 29.7$ & 0.20 .2 & $3.5,0.52$ & 6.2 & 2.1 & 1.9 & -63 & 500 \\
\hline $\mathrm{C}$ & $18: 47: 46.41$ & $-1: 54: 32.9$ & 0.20 .2 & $1.9,0.26$ & 3.7 & 2.7 & 1.7 & 63 & 300 \\
\hline $\mathrm{D}$ & $18: 47: 46.69$ & $-1: 54: 32.3$ & 0.20 .2 & $1.1,0.15$ & 1.9 & 2.1 & 1.5 & -69 & 150 \\
\hline $\mathrm{E}$ & $18: 47: 47.05$ & $-1: 54: 31.0$ & 0.20 .2 & $1.2,0.16$ & 1.6 & 1.7 & 0.9 & 28 & 130 \\
\hline $\mathrm{F}$ & $18: 47: 46.55$ & $-1: 54: 23.1$ & 0.10 .2 & $0.7,0.08$ & 1.4 & 3.4 & 0.9 & 22 & 110 \\
\hline
\end{tabular}

Note. All masses estimated using a temperature of $25 \mathrm{~K}$; core A used 25 and $300 \mathrm{~K}$.

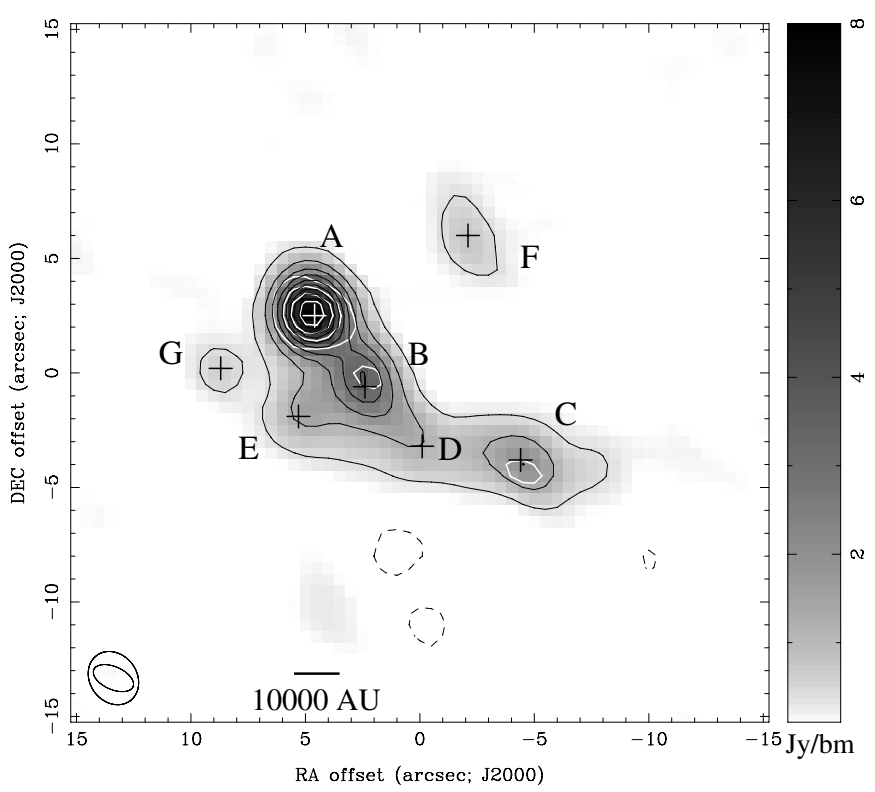

Figure 1. Gray scale image and the black contours show the $345 \mathrm{GHz}$ continuum emission. Continuum peaks brighter than the level of the highest negative contour were fitted and labeled $\mathrm{A}-\mathrm{G}$ (listed in Table 1). The $\mathrm{CH}_{3} \mathrm{CN}$ emission integrated over the first four $\mathrm{K}$-components is shown as white contours. The continuum contour levels start at $-0.4 \mathrm{Jy}_{\text {beam }}{ }^{-1}$ with a step size of $0.8 \mathrm{Jy} \mathrm{beam}^{-1}, 1 \sigma$ being $0.1 \mathrm{Jy}$ beam $^{-1}$. The line contours start at $37.5 \mathrm{Jy} \mathrm{beam}^{-1} \mathrm{~km} \mathrm{~s}^{-1}$ with a step size of $75 \mathrm{Jy} \mathrm{beam}^{-1} \mathrm{~km} \mathrm{~s}^{-1}, 1 \sigma$ being $7 \mathrm{Jy} \mathrm{beam}^{-1} \mathrm{~km} \mathrm{~s}^{-1}$. The synthesized beams are shown at the bottom left (smaller beam- $\mathrm{CH}_{3} \mathrm{CN}$ ).

\section{CONTINUUM EMISSION AND MAGNETIC FIELD}

The continuum emission, mapped using the combined data from the two configurations at a beam size of 2 ."5 $\times 2$ ". 1 shows multiple peaks (Figure 1) with integrated fluxes and masses in the range $1-10$ Jy and $100-1000 M_{\odot}$ (Table 1$)$. All the parameters of the cores were obtained by Gaussian fitting and deconvolution. The masses were estimated following Hildebrand (1983), using a dust absorption coefficient of $2 \mathrm{~cm}^{2} \mathrm{~g}^{-1}$ at $343 \mathrm{GHz}$ (Ossenkopf \& Henning 1994), a gas-to-dust ratio of 100 , and a temperature of $25 \mathrm{~K}$ from spectral energy distribution (SED) fitting (Bally et al. 2010) for all cores except the hot core A (see Section 4), where a range of $25 \mathrm{~K}-300 \mathrm{~K}$ was used.

The continuum emission shows polarization at the level of $0.5 \%-15 \%$, exhibiting the well-known polarization hole phenomenon, with the lowest polarization fractions occurring at the highest Stokes $I$ intensities. Assuming that the polarization is due to magnetically aligned dust grains, the derived $B$-field orientations are shown in Figure 2. The orientation of the polarization shows an ordered pattern, consistent with previous measurements (Cortes \& Crutcher 2006) while reaching a factor

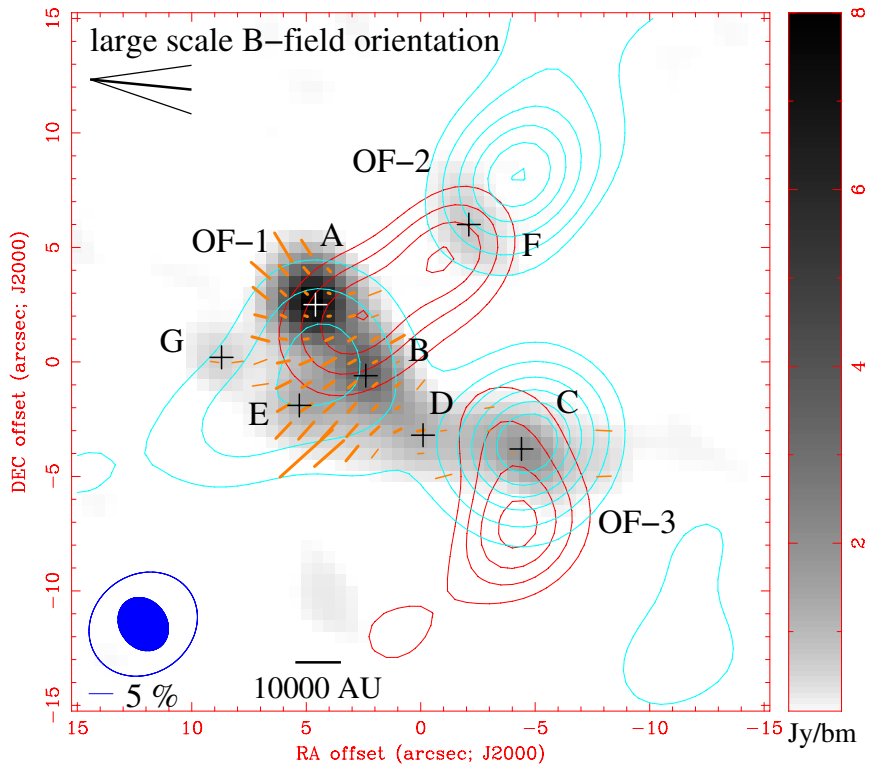

Figure 2. Continuum emission, $B$ field, and $\mathrm{CO}$ outflows. The $\mathrm{CO}$ contours start at $40 \mathrm{Jy} \mathrm{beam}^{-1} \mathrm{~km} \mathrm{~s}^{-1}$ and the step size is $40 \mathrm{Jy}_{\text {beam }}{ }^{-1} \mathrm{~km} \mathrm{~s}^{-1}, 1 \sigma$ being $10 \mathrm{Jy}$ beam $^{-1} \mathrm{~km} \mathrm{~s}^{-1}$. The $B$-field orientations are shown as line segments with lengths proportional to fractional polarization. Thick and thin segments mark $>3 \sigma$ and $2 \sigma-3 \sigma$ measurements, respectively. The orientation of the large-scale $B$ field and its range are also shown. The synthesized beams are shown at the bottom left (filled-continuum).

(A color version of this figure is available in the online journal.)

Table 2

Polarization and Outflow Position Angles

\begin{tabular}{lrrrc}
\hline \hline Region & $\begin{array}{c}\text { P.A.mean } \\
(\mathrm{deg})\end{array}$ & $\begin{array}{c}\sigma_{\text {P.A. }} \\
(\mathrm{deg})\end{array}$ & $\begin{array}{c}\delta \phi \\
(\mathrm{deg})\end{array}$ & $\begin{array}{c}\text { Number of } \\
\text { pixels }\end{array}$ \\
\hline A & -39 & 14 & 12 & 13 \\
$\mathrm{~B} / \mathrm{E}$ & 29 & 17 & 15 & 45 \\
C/D & 5 & 8 & 3 & 9 \\
All & 13 & 31 & 30 & 67 \\
Large scale $^{\mathrm{a}}$ & 175 & \pm 14 & & \\
OF-1 & 136 & & & \\
OF-2 & 135 & & & \\
OF-3 & 173 & & & \\
\hline
\end{tabular}

Note. ${ }^{\text {a }}$ Dotson et al. 2010.

of two better resolution. It changes significantly over the map and can be decomposed into three regions corresponding to the dust peaks A, B-E, and the much weaker C-D. The position angles are approximately perpendicular to each other between A and B-E. The statistics of the position angles are presented in Table 2. The measurements include 50 detections with $3 \sigma$ or better signal-to-noise ratio and 17 with $2 \sigma$. The intrinsic 


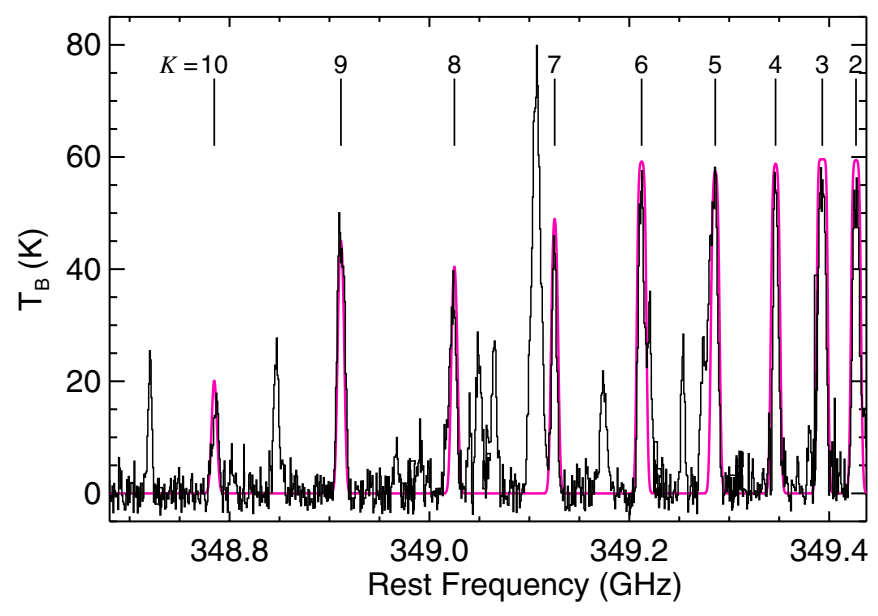

Figure 3. Spectrum and fits for the $\mathrm{CH}_{3} \mathrm{CN}(19-18), K=2$ to $K=10$ lines. The $K=0$ and 1 components are not shown. The spectrum was extracted over one synthesized beam at the peak. Other prominent lines seen in this spectrum are tentatively identified to be from $\mathrm{CH}_{3} \mathrm{OH}(349.107 \mathrm{GHz}), \mathrm{CH}_{2} \mathrm{CHCN}$ (348.991 GHz), $\mathrm{HCOOCH}_{3}(349.048$ and $349.066 \mathrm{GHz}), \mathrm{CH}_{3}^{13} \mathrm{CN}(349.253$ and $349.173 \mathrm{GHz})$, and $\mathrm{C}_{2} \mathrm{H}_{5} \mathrm{OH}(348.848$ and $348.720 \mathrm{GHz})$.

(A color version of this figure is available in the online journal.)

dispersion of the position angle of the polarization, $\delta \phi$, is calculated by subtracting in quadrature an average position angle measurement error of 7.5, arising from a $5 \mathrm{mJy}$ rms on the $\mathrm{Q}$ and $\mathrm{U}$ images, from the observed rms of the position angles, $\sigma_{\text {P.A. }}$.

For the region $\mathrm{B}-\mathrm{E}$, with the most numerous data points, we estimate the strength of the $B$ field using the Chandrasekhar-Fermi method. Following Crutcher et al. (2004),

$$
B_{\text {pos }}=9.3 \times n\left(\mathrm{H}_{2}\right)^{0.5} \delta V / \delta \phi,
$$

where $B_{\text {pos }}, n\left(H_{2}\right), \delta V$, and $\delta \phi$ are the plane-of-sky $B$ field $(\mu \mathrm{G})$, density $\left(\mathrm{cm}^{-3}\right)$, FWHM of the turbulent velocity dispersion $\left(\mathrm{km} \mathrm{s}^{-1}\right.$ ) and the $B$-field position angle dispersion (degree), respectively. We estimate $n\left(\mathrm{H}_{2}\right)$ to be $10^{7} \mathrm{~cm}^{-3}$ by combining the masses of cores $\mathrm{B}$ and $\mathrm{E}$, and a size of $5^{\prime \prime}$. For $\delta V$, we use a value of $3 \mathrm{~km} \mathrm{~s}^{-1}$ from the single dish $\mathrm{HCO}^{+}$measurement of Cortes et al. (2010) with a $20^{\prime \prime}$ beam, thus avoiding any interferometric spatial filtering. The $\mathrm{CH}_{3} \mathrm{CN}$ linewidth, on a much smaller $\sim 1^{\prime \prime}$ spatial scale, is still similar at $5 \mathrm{~km} \mathrm{~s}^{-1}$ (Section 4), and we consider the single dish measurement to be more representative of the velocity dispersion over the $\sim 7^{\prime \prime}$ region over which the position angle dispersion is measured. The resulting $B_{\text {pos }}$ is $6 \mathrm{mG}$. The mass-to-flux ratio estimated from these numbers is 3.5 , and $\sim 1$ when a statistical geometric correction is applied, implying a critical to slightly super-critical condition. This is consistent with previous measurements (Cortes \& Crutcher 2006; Cortes 2011) and would suggest that similar conditions are maintained on smaller spatial scales. Nevertheless, we caution that these estimates are subject to large uncertainties.

\section{HOT CORE}

Strong emission from several hot core species was detected toward core A. Cores B and $\mathrm{C}$ also show emission at much weaker levels. Here, we focus on the remarkable $\mathrm{CH}_{3} \mathrm{CN}(J=$ 19-18) emission from core A, where $11 \mathrm{~K}$-ladder components were detected. The integrated emission and spectrum are shown in Figures 1 and 3, respectively. This data set only consists of the compact configuration observations and has a beam size of 2 .' $1 \times 1^{\prime \prime}$. The emission is marginally resolved with a source size of $1^{\prime \prime} .5 \times 00^{\prime \prime} .6(8000 \times 3000 \mathrm{AU})$. The detection of a large number of lines from the K-ladder suggests a high temperature, the energy level for the $K=10$ line being $885 \mathrm{~K}$. The lowest $\mathrm{K}$ lines are optically thick, as seen from their similar strengths. Assuming all the $\mathrm{K}$ components are in LTE and trace the same gas, a grid search $\chi^{2}$ minimization was used to fit for temperature, column density, and source size, including optical depth effects (Qiu \& Zhang 2009). Using the $K=2-10$ components (Figure 3 ), we get $300 \mathrm{~K}, 2 \times 10^{17} \mathrm{~cm}^{-2}$, and 0.6 (3000 AU) for the temperature, column density, and source size. Fitting only for the optically thin high $\mathrm{K}$ components 7-10, the values are $420 \mathrm{~K}, 4 \times 10^{16} \mathrm{~cm}^{-2}$, and $1^{\prime \prime} .2$. A systemic velocity of $101.5 \mathrm{~km} \mathrm{~s}^{-1}$ was determined and the line width was set at $5 \mathrm{~km} \mathrm{~s}^{-1}$ guided by Gaussian fits to the $K=3,4$, and 9 components, which appear to be free from blending, and the quality of the fit to the ladder. Such high temperatures are uncommon and only seen in a few other cases, some examples being Orion BN/KL ( $400 \mathrm{~K}$; Wilson et al. 1993; Goddi et al. 2011) and W51 IRS2 ( 310 K; Mauersberger et al. 1987). For comparison, the source size and luminosity can be used to obtain an independent estimate of the temperature. Taking the luminosity from SED fits of $3 \times 10^{4} L_{\odot}$ (Bally et al. 2010; Herpin et al. 2012), following Scoville \& Kwan (1976; also Scoville 2012), a dust temperature of 70-120 K (depending on opacity) is obtained for a radial distance of $2500 \mathrm{AU}$ (geometric mean of the semimajor and semiminor axes), assuming heating by stellar radiation. The Herpin et al. model also shows a lower temperature $(\sim 150 \mathrm{~K}$; as shown in their Figure 5$)$ for the $2500 \mathrm{AU}$ distance. If this dust temperature also characterizes $\mathrm{CH}_{3} \mathrm{CN}$ excitation (Doty et al. 2002), its disagreement with the measured temperature of $300-400 \mathrm{~K}$ points to the possibility that stellar heating alone may not be sufficient to account for the hot $\mathrm{CH}_{3} \mathrm{CN}$. More detailed modeling and corroboration from other data will be needed for further clarity. In summary, we suggest that a high-mass proto-cluster may be forming here within a compact $\sim 0.01 \mathrm{pc}$ radius region, although we do not yet have evidence for multiplicity.

\section{OUTFLOWS}

The outflows are mapped and studied using CO (3-2) emission. The CO data only consist of sub-compact array observations and thus have a poorer spatial resolution of $5^{\prime \prime}$. The CO line shows emission to large $\left(\sim \pm 70 \mathrm{~km} \mathrm{~s}^{-1}\right)$ velocities. In Figure 2, we present integrated emission over the velocity ranges $60-90$ and $120-165 \mathrm{~km} \mathrm{~s}^{-1}$, with the systemic velocity based on our $\mathrm{CH}_{3} \mathrm{CN}$ data being $101.5 \mathrm{~km} \mathrm{~s}^{-1}$. The $\mathrm{CO}$ emission appears to trace three bipolar outflows, two of which are associated with the cores $\mathrm{C}$ and $\mathrm{F}$ (OF-3 and OF-2, respectively) and the third is centered between cores $\mathrm{A}$ and $\mathrm{B}(\mathrm{OF}-1)$. Higher resolution observations will be needed to clarify the location of the driving source for OF-1. Outflows 1 and 2 are oriented the same way but with opposite red and blue lobes. The velocity ranges used were chosen to best delineate the outflows. We estimate outflow masses for the three outflows following the approach in Garden et al. (1991). Assuming optically thin emission the outflow masses derived are 12,11 , and $14 M_{\odot}$ for OF-1, 2, and 3, respectively, for an excitation temperature of $20 \mathrm{~K}$. Based on the single dish $\mathrm{CO}(3-2)$ brightness temperature from Cortes et al. (2011), $20 \mathrm{~K}$ was taken to be the lower limit and the mass estimate is not very sensitive to temperature, staying within a factor of two for temperatures up to $180 \mathrm{~K}$. The massive outflows imply high-mass star formation. The outflows 
have time scales of $\sim 10^{4} \mathrm{yr}$, estimated using outflow extents and velocities of $5^{\prime \prime}(\sim 0.1 \mathrm{pc})$ and $25 \mathrm{~km} \mathrm{~s}^{-1}$, pointing to their youth.

\section{SUMMARY AND DISCUSSION}

Our observations show multiple high-mass star formation in the W43-MM1 region. A compact hot core detected in a number of spectral lines has a temperature of $\sim 400 \mathrm{~K}$, derived from $\mathrm{CH}_{3} \mathrm{CN}$ emission. This high temperature can help in the investigation of high temperature chemistry probed by other spectral lines being studied (not included in this Letter). Multiple massive outflows were mapped in CO. Dust polarization measurements over the main outflow show an alignment between the outflow and the $B$-field orientation on the plane of the sky.

We now seek to compare our results with the measurements of the larger scale $B$ field reported by Dotson et al. (2010). These single dish measurements obtained with the CSO/Hertz have a beam size of $\sim 18^{\prime \prime}$. While the position angles for the six detections reported are spread over $\sim 90^{\circ}$, a range similar to our small scale measurements, the innermost four measurements are well ordered, nearly parallel to each other. The outermost two are nearly perpendicular to each other. We exclude the outer two measurements and take the average of the four closest measurements to define the "large scale" field, oriented at a position angle of $85^{\circ}\left(175^{\circ}\right.$ for polarization) with a range of $\pm 14^{\circ}$. This "large scale" corresponds to $\sim 40^{\prime \prime}(\sim 1 \mathrm{pc})$ and the SMA measurements are on a $\sim 7^{\prime \prime}(\sim 0.1 \mathrm{pc})$ scale. It is possible to exclude only the most deviant single dish measurement, in which case the remaining five measurements trace a gradually changing pattern with the innermost four measurements defining the larger scale field for comparison. As seen in Figure 2, the field orientation over the main outflow (OF-1) is not parallel to this large scale field measured from the single dish observations. This suggests the possibility that the magnetic fields, as seen on the small scales, changed orientation from a nearly parallel distribution over the immediate larger scale neighborhood either during the formation of the cores or because the star formation activity influenced the present morphology.

To assess the possibility of the outflow influencing the $B$ field morphology, we compare the total mechanical energy in the outflow with the energy in the magnetic field, noting that the outflow extent and the region over which the $B$ field is mapped are comparable. We estimate the outflow energy to be $\sim 10^{47} \mathrm{erg},\left(=M v^{2}\right)$, taking an outflowing mass, $M$, of $10 M_{\odot}$ with a velocity, $v$, of $25 \mathrm{~km} \mathrm{~s}^{-1}$. The magnetic energy calculated is also $\sim 10^{47} \mathrm{erg}$, considering the volume over which the $B$ field is measured to be a cylinder of length $l=4 \times 10^{4} \mathrm{AU}\left(7^{\prime \prime}\right)$ and diameter $2 \times 10^{4} \mathrm{AU}\left(4^{\prime \prime}\right)$, as seen in the map for the B-E region, and a magnetic energy density of $B^{2} / 8 \pi=2 \times 10^{-6} \mathrm{erg} \mathrm{cm}^{-3}$, using the value of $B$ from Section 3 . As these energies are similar, based on these numbers it is not possible to say if the outflow influenced the orientation of the $B$ field. Given the uncertainties in these estimates, we would need a large difference to be able to make a more definitive statement. While the comparison is inconclusive, we note that although the outflow mapped in $\mathrm{CO}$ traces densities of $\sim 10^{3} \mathrm{~cm}^{-3}$ and the $B$ field is measured by polarization originating in a much denser medium $\left(\sim 10^{7} \mathrm{~cm}^{-3}\right)$, it is still appropriate to compare the energies of the two. This is because the outflow has cleared a lower density cavity in the dense medium into which it was originally driven and it could have influenced the magnetic field during its early phase. In addition, magnetic tension can couple the magnetic fields in different regions. If alignment is caused by the outflow, then the field strength cannot be estimated using the C-F method. However, the estimate in Section 5 is still a measure of the upper limit to the $B$ field, as the position angle dispersion resulting from turbulence is reduced by the outflow-induced alignment. Thus, the estimates above are only to be taken as indicative of the strength of the $B$ field.

There are some studies of alignment between outflows and $B$ fields in the literature, but the results are inconclusive (see $\mathrm{Li}$ et al. 2014 for a review and discussion; Curran \& Chrysostomou 2007; Wolf et al. 2003; Davidson et al. 2011; Chapman et al. 2013; Hull et al. 2013). They are predominantly single dish observations toward low-mass, star-forming regions, except for the Hull et al. study, which used the CARMA interferometer. There is a clear conflict between the results of the most recent Chapman et al. and the Hull et al. studies, the first showing alignment between the $B$ field and outflow orientations, and the second showing no correlation. As suggested by Chapman et al. (see also Li et al. 2014), the two may be reconciled by the fact that they trace different scales dominated by different processes. Our data correspond to the spatial scales of the Chapman et al. study ( $10,000 \mathrm{AU})$ where an alignment is seen. However, a simple global picture - a strong magnetic field on large scales directing collapse along its orientation, leading to the formation of flattened pseudo-disk structures, and rotation axes aligned to the $B$ field by magnetic breaking and the alignment of outflows to this axis-is inconsistent with our observations. This is because (1) the field orientation in our map varies on small scales, and one of the three outflows is not aligned to the other two, and (2) the large scale field is not aligned to either of the directions of the outflows or to the small scale $B$ field. Observations with finer spatial resolutions can help us delineate potential pseudo-disk structures and study their rotation and relationship to the outflows and $B$-field orientations. The presence of multiple outflows within a small region presents a good opportunity to pursue this avenue.

Comments from an anonymous referee which helped improve the Letter are gratefully acknowledged.

\section{REFERENCES}

Bally, Anderson, L. D., Battersby, C., et al. 2010, A\&A, 518, 90 Beuther, H., Schilke, P., Sridharan, T. K., et al. 2002, A\&A, 383, 392 Beuther, H., Vlemmings, W. H. T., Rao, R., \& van der Tak, F. F. S. 2010, ApJL, 724, L113

Cesaroni, R., Hofner, P., Araya, E., \& Kurtz, S. 2010, A\&A, 509, 50

Cesaroni, R., Walmsley, C. M., \& Churchwell, E. 1992, A\&A, 256, 618

Chapman, N., Davidson, J., Goldsmith, P., et al. 2013, ApJ, 770, 151

Cortes, P. 2011, ApJ, 743, 194

Cortes, P., \& Crutcher, R. 2006, ApJ, 639, 965

Cortes, P. C., Crutcher, R. M., Shepherd, D. S., \& Bronfman, L. 2008, ApJ, 676,464

Cortes, P. C., Parra, R., Cortes, J. R., \& Hardy, E. 2010, A\&A, 519, 35

Crutcher, R. M., Nutter, D. J., Ward-Thompson, D., \& Kirk, J. M. 2004, ApJ, 600,279

Curran, R. L., \& Chrysostomou, A. 2007, MNRAS, 382, 699

Davidson, J., Novak, G., Matthews, T., et al. 2011, ApJ, 732, 97

Dotson, Vaillancourt, J. E., Kirby, L., et al. 2010, ApJS, 186, 406

Doty, S. D., van Dishoeck, E. F., van der Tak, F. F. S., \& Boonman, A. M. S. 2002, A\&A, 389, 446

Garden, R. P., Hayashi, M., Hasegawa, T., Gatley, I., \& Kaifu, N. 1991, ApJ, 374,540

Girart, J. M., Beltrán, M., Zhang, Q., Rao, R., \& Estalella, R. 2009, Sci, 324,1408

Girart, J. M., Frau, P., Zhang, Q., et al. 2013, ApJ, 772, 69

Goddi, C., Greenhill, L. J., Humphreys, E. M. L., Chandler, C. J., \& Matthews, L. D. 2011, ApJ, 739, 13 
Herpin, F., Chavarría, L., van der Tak, F., et al. 2012, A\&A, 542, 76 Hildebrand, R. H. 1983, QJRAS, 24, 267

Ho, P. T. P., Moran, J. M., \& Lo, K. Y. 2004, ApJL, 616, L1

Hull, C., Plambeck, R., Bolatto, A., et al. 2013, ApJ, 768, 159

Lai, S.-P., Girart, J. M., \& Crutcher, R. M. 2003, ApJ, 598, 392

Li, H., Goodman, A., Sridharan, T. K., et al. 2014, Protostard and Planets VI, in press

Marrone, D. P., Moran, J. M., Zhao, J.-H., \& Rao, R. 2006, ApJ, 640, 308

Marrone, D. P., \& Rao, R. 2008, Proc. SPIE, 7020, 70202B

Mauersberger, R., Henkel, C., \& Wilson, T. L. 1987, A\&A, 173, 352

Motte, F., Schilke, P., \& Lis, D. C. 2003, ApJ, 582, 277

Nguyen-Lu'o'ng, Q., Motte, F., Carlhoff, P., et al. 2013, ApJ, 775, 88

Ossenkopf, V., \& Henning, Th. 1994, A\&A, 291, 953

Qiu, K., \& Zhang, Q. 2009, ApJ, 702, L66
Scoville, N. Z. 2012, in Secular Evolution of Galaxies, ed. J. Falcon-Barroso \& J. H. Knapen (Cambridge: Cambridge Univ. Press), 491

Scoville, N. Z., \& Kwan, J. 1976, ApJ, 206, 718

Shepherd, D., \& Churchwell, E. 1996, ApJ, 472, 225

Sridharan, T. K., Beuther, H., Schilke, P., Menten, K. M., \& Wyrowski, F. 2002, ApJ, 566, 931

Tang, Y., Ho, P. T. P., Koch, P., Guilloteau, S., \& Dutrey, A. 2013, ApJ, 763,135

Tang, Y.-W., Ho, P. T. P., Girart, J. M., et al. 2009a, ApJ, 695, 1399

Tang, Y.-W., Ho, P. T. P., Koch, P. M., et al. 2009b, ApJ, 700, 251

Wilson, T. L., Henkel, C., Huttemeister, S., et al. 1993, A\&A, 276, L29

Wolf, S., Launhardt, R., \& Henning, T. 2003, ApJ., 592, 233

Zhang, B., Moscadelli, L., Sato, M., et al. 2014, ApJ, 781, 89

Zhang, Q., Hunter, T. R., Brand, J., et al. 2005, ApJ, 625, 864 